2 Corresponding author:

3 Alan Fleming, Australian Maritime College | NCMEH, University of Tasmania,

4 Swanson Building, B11, Launceston Tasmania, 7250, Australia

5 Email: Alan.Fleming@utas.edu.au

6 Application of photogrammetry for 7 spatial free surface elevation and velocity 8 measurement in wave flumes

$9 \quad$ Alan Fleming ${ }^{1}$, Brian Winship ${ }^{1}$, Gregor Macfarlane ${ }^{1}$

$10{ }^{1}$ Australian Maritime College, University of Tasmania, Tasmania, Australia

\title{
12 Abstract
}

13 This paper presents a method for obtaining the spatial free-surface elevation and velocity

14 field for the water surface in a wave flume over a relatively large measurement area for

15 this type of application (approximately $1.5 \mathrm{~m} \mathrm{x} 1.5 \mathrm{~m}$ ). The technique employs

16 proprietary videogrammetry software to post-process stereo images captured by multiple

17 synchronised machine vision cameras. Dimensional resolution and other limitations are

18 similar to that experienced for Particle Imaging Velocimetry (PIV) systems $(x, y$

19 resolution of $2 \mathrm{~mm}$ ). Imaging of the free surface was enabled by the use of millions of bespoke slightly positively buoyant fluorescent flakes. Ultraviolet light (UV) was used as the primary light source to excite the fluorescent flakes. Reflected UV light was attenuated by a high-pass filter fitted to the cameras so that only the emitted light from the fluorescent flakes was visible.

24 The software was validated using a simple linear translation experiment. An application is demonstrated for the radiated wave field generated from a submerged sinusoidal heaving sphere for two cases: one single and five consecutive oscillations. Results agree with linear wave theory which indicates that the floating flakes had minimal impact on the water surface particle motion at the scale tested.

29 It is therefore concluded that spatial measurement of the free-surface elevation and velocity using the method presented has good resolution over a large measurement field.

31 The flakes were found to follow the free-surface well, but the measurement area is 32 constrained to where the pattern of flakes exists in the image. Hence, application of 33 floating markers is not suitable for experiments with significant outflow/upwelling which

34 would wash away the floating markers from the intended measurement area. 
2 Digital Image Correlation, Videogrammetry, wave flume, surface flow, experimental,

3 velocity field, spatial free surface measurement.

Accurate measurement of water surface elevation in wave flumes and towing tanks is fundamental to experiments performed in those facilities. Commonly employed single point surface elevation measurement techniques include contact measurement (float/water surface following, resistive/capacitive style wave probes) and non-contact methods (ultrasonic and optical) ${ }^{1}$ or indirect methods such as the laser slope gauge ${ }^{2}$. The single point measurement systems are generally regarded as accurate (sub-mm accuracy) when properly calibrated.

The single point measurement techniques can be extended for spatial measurement of surface elevation by using arrays of the single point devices. For example Stratigaki ${ }^{3}$ had an array consisting of 41 resistance type wave probes to measure intra-array effects between scale model wave energy converters while Fleming et al. ${ }^{4}$ employed an array of five wave probes to measure the free-surface in an oscillating water column. More recently O'boyle et al..$^{5}$ implemented a linear array of 32 traversing wave probes utilising repeat experiments to measure a 2D wave field water elevation in pseudo irregular seas. The spatial resolution of these types of systems is constrained by electrical and physical interference between individual probes which typically means a minimum spacing in the range of tens of millimetres is possible. Direct measurement of surface particle velocity fields by these sensors is not possible and the cost of an array of sensors is directly proportional to the number of sensors in the array, so it soon becomes inhibitive for arrays of tens of sensors, plus the infrastructure and time required to position and calibrate them. It should also be noted that there have been significant advancements in development of non-contact 2D Light Imaging and Range Detection systems ${ }^{6,7}$ for water surface elevation measurement, but to date they still offer lower accuracy and reliability than conventional contact based methods.

In recent years various image based systems have been developed and implemented at various scales for the spatial and temporal measurement of water surface elevation ${ }^{2,8-14}$, particle velocity ${ }^{15-17}$, or both simultaneously ${ }^{18-20}$. One way to categorise these imaging based methods for spatial surface measurement is by considering the different properties of light at the interface of two different fluids ${ }^{8}$ (air and water). The main phenomena exploited include; direct observation (emission), specular reflection (off the water surface), and refraction of light through the air/water interface. Generally each method exploits only one of those optical properties, where the presence of other objects appearing as image processing artefacts diminish the quality of the calculated result. For example; most methods that directly image the free-surface require quality images of a textured free-surface or distinct particles. Any presence of light as specular reflection or refraction (for example; visible objects below the free-surface) will diminish the solved surface compared to similar data which does not contain the unwanted artefacts. 
Direct imaging based techniques can be considered the most advanced and robust of these methods, which in part is due to the wider adoption of the methodology. Typical methods include digital image correlation (photogrammetry) ${ }^{9,21-23}$, Particle Imaging Velocimetry (PIV) ${ }^{15,18,19}$, and Particle Tracer Velocimetry (PTV) ${ }^{16,20,24}$. Commercially, there are several turn-key systems available for capturing and processing data of this type. However implementation of any of these systems for use in hydrodynamic facilities will have similar technical challenges in directly imaging the free-surface. Arguably the main technical challenge is to minimise the presence of specular reflection and unwanted visible objects below the free-surface. The free-surface in hydrodynamic facilities such as towing tanks and wave flumes may also contain insufficient features for satisfactory image correlation without additional treatment ${ }^{8}$.

Turney et al. ${ }^{19}$ overcame reflection and refraction imaging problems to measure interfacial particle velocities of 40 micron fluorescent seeding particles in a wind wave flume by rendering the water opaque with dye and use of a 'blue light', thereby imaging only the near-surface fluorescent seeding particles. The method requires the entire experimental volume to be dyed and seeded, so is only feasible in modestly sized facilities such as the $2.5 \mathrm{~m}^{3}$ of that study.

In the field of full-scale wave measurements some groups have recently improved stereo videogrammetry algorithms to identify and compensate for specular reflection, including the release of an open source software referred to as Wave Acquisition Stereo System (WASS) ${ }^{9}$. The WASS software has been shown to be effective for full scale measurement of water waves in a large measurement area $(30 \mathrm{~m} \mathrm{x}$ $30 \mathrm{~m})^{22}$. The algorithm to deal with specular reflection relies on the Lambertian assumption, so is most reliable with diffused light ${ }^{2}$, which also requires the cameras to be mounted with a parallel viewing axis. Zavadsky et al. ${ }^{2}$ applied WASS in a small wind wave flume for a measurement area of $0.25 \mathrm{~m} \mathrm{x} 0.40 \mathrm{~m}$ and reported that measurements had more noise than a wave gauge, but found that wave statistical results were similar. They also emphasised the difficulty in producing appropriate illumination in a closed laboratory.

Methods have been developed based on specular reflection of an image projected on the water surface ${ }^{13,25}$. Kiefhaber et al..$^{25}$ used specular reflection to their advantage in a novel stereo imaging approach where two cameras were mounted within infrared LED arrays and focused on the same region. Their system uses an inverted light path with two cameras and two UV LED arrays, where each camera is mounted in an LED array. Each camera and matching LED array have the same light axis and are angled toward the measurement area so the alternate camera and UV LED array are in the nominal path of the reflected light. Each camera is sampled in turn while the alternate LED array is illuminated. In this way an inverted light path is formed. The method was shown to be reliable for a measurement area of $30 \times 20 \mathrm{~cm}$, but scaling to large measurement areas would be subject to illumination/light source complications and the occurrence of dropout (absence of reflections) would increase.

Systems that utilise refraction of light at the free-surface indirectly measure the water surface elevation by first measuring the water surface slope and then estimating the 
elevation from wave theory. Generally, the methods place the camera above the water surface and image a target on the tank bottom. Moisey et al ${ }^{10}$ describe a Schlieren method which utilise a single camera system directly over a submerged array target. The method is inexpensive to implement and well suited for non-intrusive measurement of small waves but is unable to resolve strong curvature and only applies to weak deformations ${ }^{10}$, furthermore the method requires a clear optical path to the tank floor. The same method was later applied by Damiano et al. ${ }^{11}$ to investigate a bouncing droplet, which demonstrates the usefulness of the system for non-intrusive and inexpensive small scale experiments. Aureli et al. ${ }^{14}$ utilise co-located colour and infrared CCD sensors with a dichromatic mirror to provide co-registered images to measure slope of the air-water interface. The author's state that the method may easily be extended to larger facilities since the method does not require a telecentric system; however, a backlit checkerboard target must be placed at or below the tank bottom. Engelen et al. ${ }^{12}$ utilise a similar experimental setup, but employ a stereo camera in place of the co-registered cameras. Gomit et al. ${ }^{26}$ measured spatial elevations of ship wake with a stereo PIV like setup with two cameras mounted above the free-surface as a stereo pair and a third camera below the free-surface to provide a reference image, the water was seeded with typical PIV tracer particles. Extension of PIV based systems to large measurement areas is generally considered not feasible since the laser power is normally a limiting factor for field of view size in PIV based experiments.

Image based measurement of the free surface in larger experimental facilities, where the measurement area is defined in the order of metres rather than centimetres, is a non-trivial task. In such facilities the water is typically clean (transparent) and the surface is a specular reflector. A photograph taken of these facilities will usually show only the floor of the tank and reflection from one or more light sources but very little water surface will be visible. Even if it was possible to exclusively image the surface, there is likely insufficient texture in the image to enable reliable image correlation. The method we apply to overcome these challenges in this paper combines the ideas of fluorescent seeding and floating particles ${ }^{16,17,27}$, but we utilise weakly buoyant fluorescent wax flakes ${ }^{27}$ with a typical direct image correlation of stereo image pairs. Considering the scale of these experiments, surface particle interaction was assumed to have minimal impact on the experimental outcome.

In the methodology section we briefly describe the software, followed by a detailed description of the experimental setup, a simple verification test and quantification of bias error. In the results section we present a case study of processed surface flow data relating to the radiated wave field generated by a heaving sphere. Conclusions on the limitations and benefits of the system follow.

For free-surface measurement we utilise a typical stereo imaging setup and process data with the software DaVis 8.2, and the add-on packages StrainMaster DIC /

42 Surface flow and some features of the Stereo PIV package developed by LaVision 
GMBH. The software uses Digital Image Correlation (DIC) to identify corresponding points in stereoscopic camera image pairs to map the objectives' surface. Through a calibration process, the position of the two cameras relative to the experimental area is determined as well as some characteristics of the lens distortion. Then with an iterative Least Square Matching (LSM) algorithm equivalent pixels in the two cameras are identified, from which it is possible to deduce the 3D surface, and the relative pixel locations. Sub-pixel interpolation is achieved with cubic B-spline interpolation. The LSM algorithm is then applied to a subsequent 3D image to deduce the velocity of the particles with an affine transform. The processing uses a region grow pyramid from a user defined seeding point.

\section{Experimental setup}

The experiments reported here were conducted as part of an extensive experimental campaign within an Australian Renewable Energy Agency funded project (grant A00575). The primary objective of the project was to develop and validate a web based planning tool for use by Governments and wave energy device developers to understand the impact on performance of changing the spacing between wave energy devices. Part of the project was a campaign consisting of over 1000 experimental runs which utilised the photogrammetry method described in this paper to image the water surface around different array configurations of wave energy converter analogues. A full description of the experimental setup is not necessary here, however the interested reader can obtain further detail on the project in the following references ${ }^{28-30}$. The broader aim of the research reported here was to obtain quality water surface elevation and velocity measurements utilising publically available DIC software. It is worth noting that the open source WASS software described by Bergamasco et al. ${ }^{9}$ was only publically available after the project was finalised, but also doesn't offer surface velocity measurements.

Experiments were performed in the Australian Maritime College's Model Test Basin (MTB) which is $35 \mathrm{~m}$ long, $12 \mathrm{~m}$ wide and capable of $1 \mathrm{~m}$ depth but here was filled to a depth of $0.6 \mathrm{~m}$. A linear motor driven sphere of $250 \mathrm{~mm}$ diameter was positioned at the approximate centre of the MTB. At mid-stroke, the top of the heaving sphere was 515 $\mathrm{mm}$ above the basin floor. Infrastructure to support the linear motor in position was placed in a bespoke pit so that only the sphere and supporting post were located above the basin floor (FIG. 1 left). The purpose of the sphere was to oscillate in sinusoidal motion to radiate waves in a coherent fashion in either the heaving or surging configuration. Results presented here are limited to the heaving configuration.

The videogrammetry system consisted of two sets of machine vision cameras with overlapping fields of view to provide a larger measurement area (FIG. 1 right). Wide angle lenses were used to increase the field-of-view, as the camera positioning was restricted by the ceiling of the test facility. The cameras were positioned on a single cross beam which was supported at each end by vertically mounted stepper motor driven linear slides. This enabled easy translation of the cameras during the calibration process while maintaining relative position between the cameras. The first camera system was positioned to monitor the area directly over the sphere and consisted of two Basler Beat

43 (ACA4000) 12 bit, 12 megapixel cameras each fitted with a $24 \mathrm{~mm}$ tilt shift lens (TSE24) 
with a Birger adapter (to adjust lens aperture) and an orange longpass filter (Midopt 550 $\mathrm{nm}$ Filter M82.0x0.75). Cameras were connected to a PC fitted with two Silicon Imaging microEnable IV frame grabbers and synchronisation boards. Images were later down sampled to a resolution of 2048 x 1536 ( 3 megapixel) to enable faster post processing time while maintaining the desired resolution. The second camera pair were centred on an area offset by $-1300 \mathrm{~mm}$ in the $x$ direction and consisted of two Basler ACE (acA204090um) 12 bit, 4 megapixel USB3 cameras, each fitted with a Kowa 6 mm LM6HC, 1.5 X 8 Edmund optics lens extender and c mount orange longpass fiter (Midopt LP550-25.4).

9 Cameras were connected to a PC via $10 \mathrm{~m}$ long fibre optic USB3 extension cables.

Image acquisition was synchronised via a hardware clock at a rate of 25 frames per second. Commencement of data acquisition coincided with initiation of linear motion via a separate hardware trigger. Images for both sets of computers were copied to RAM and stored using in house software.
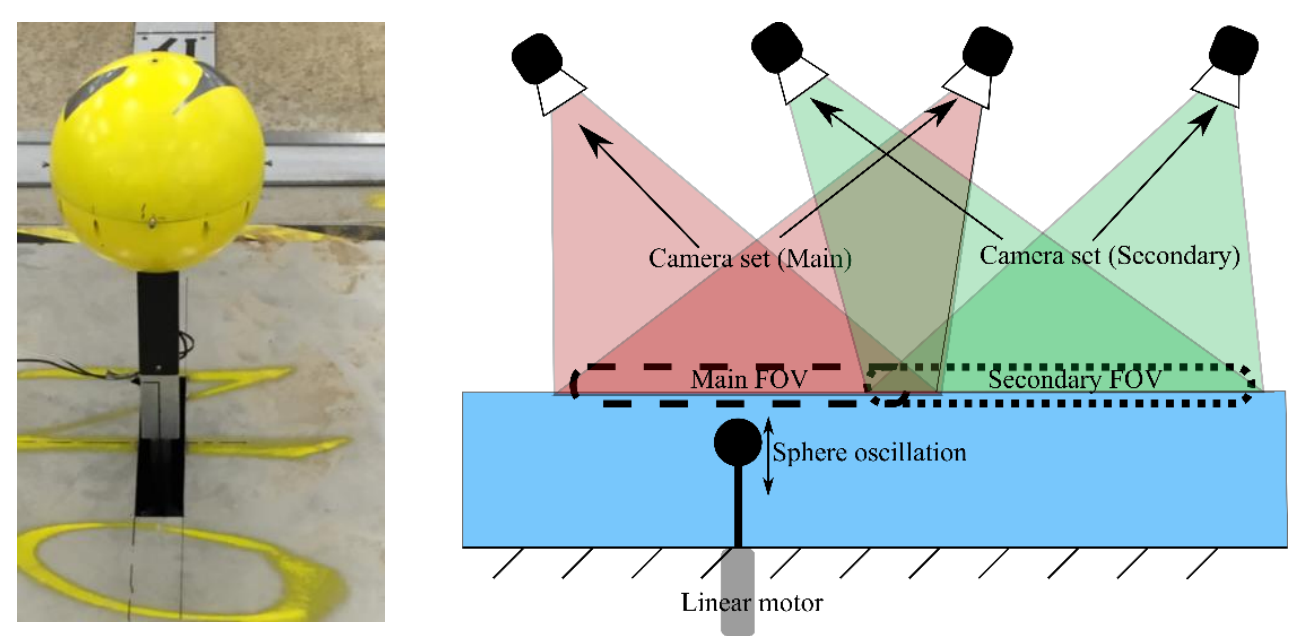

FIG. 1: Left - $250 \mathrm{~mm}$ diameter sphere on post in-situ in the MTB without water. Linear motor and support frame are hidden below the floor plate. Right: Representation of camera layout and sphere location.

\section{Surface markers}

As mentioned in the introduction, imaging of the free-surface in wave flumes and towing tanks is a non-trivial task. For this purpose, in the order of one million flakes were manufactured in house consisting of a blend of paraffin wax, carnauba wax and ultraviolet fluorescent pigment in the ratio of 90:7.5:2.5 by weight respectively. The approximate dimensions of the flakes (FIG. 2 bottom left) were $5 \mathrm{~mm}$ x $5 \mathrm{~mm}$ and $1 \mathrm{~mm}$ thick, and had specific gravity of approximately 0.93 , each flake occupied between 4 and 10 pixels of an image to produce a textured image typical as portrayed in FIG. 2 - centre. The irregularity of the flakes was sufficient to provide the texture necessary for the photogrammetry software. A high coverage factor was found to give best processing results as the flakes were weakly attracted to one another. Once the flakes were fully 
wetted, after approximately three days of immersion in water, almost the entire flake was submerged with only the occasional corner of flakes breaching the surface, or where

3 particles physically overlapped.

A floating fence was used to contain and concentrate the wax flakes within a roughly rectangular area of approx. $7 \mathrm{~m}$ x $6 \mathrm{~m}$ meaning approximately $40 \mathrm{~kg}$ of flakes were required. The fence was fabricated from $100 \mathrm{~mm}$ wide strips of $3 \mathrm{~mm}$ thick closed cell foam with evenly spaced clumped lead weights used to provide a suitable righting moment to keep the fence vertically aligned (FIG. 2 left top and right). Position of the 8 fence was maintained by generating a restoring force through 12 separate vertical nylon 10 lines equally spaced around the fence. Each nylon line passed over a pulley connected to one of two overhead trusses positioned over the front and rear of the area of interest. A clump weight was suspended in air from each nylon line to generate the restoring force such that the attachment point of the nylon line on the fence was directly below the pulley. Ballasting of the fence was adjusted to minimise interference of the fence on the generated wave fields and was found to be relatively transparent.

Although results are not provided on experiments utilising the in-built wave generator in the MTB in this paper, it is worth mentioning that the fences were observed to be highly transparent to long-crested waves, the fence would move and deform under the wave action with the nearby water particle motion with minimal radiation or absorption of the incoming wave. For short-crested waves the fence drifted down-wave with the water particle displacement related to the action of Stokes' drift, thus limiting experimental time to approximately 40 seconds as the clumped weights reached the extent of their travel (which caused significant interference to the incoming wave field).

Excitation of the fluorescent wax flakes was achieved with a total of 12 ultraviolet stage wash lights (each consisting of an array of 54 X 3 Watt UV LEDs). The stage lights were mounted on two overhead trusses and directed toward the ceiling over the area of interest which was found to diffuse the light effectively to provide a sufficiently uniform light signal throughout the field of view for both sets of cameras. Diffused and consistent light intensity was found to improve image processing and also enables use of a lower image sensor bit depth without saturation.
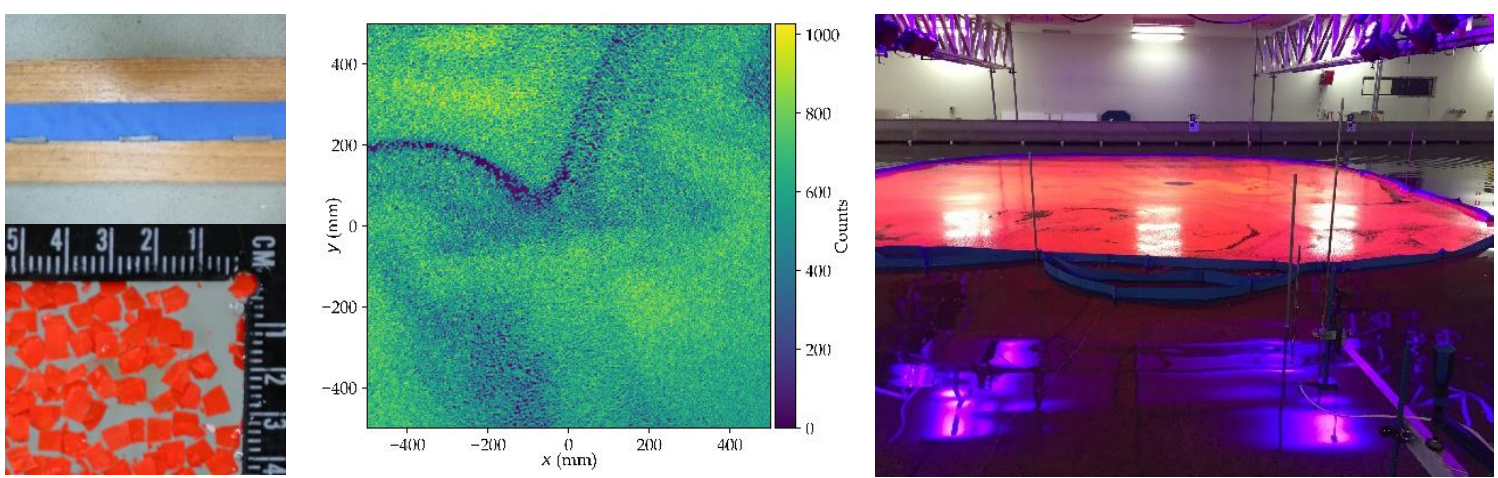
1 FIG. 2: Left top: A small section of the (blue) floating fence laid out flat with three lead 2 weights on the lower edge. Left bottom: Close up of flakes floating on the free surface 3 (rule scale in $\mathrm{cm}$ ) Centre: A section of a deformed (corrected) image of the free surface.

4 Right: UV excited fluorescent flakes held in station over the measurement area contained 5 by the floating fence.

6 Calibration

The calibration process employs a calibration plate and software wizard to derive 8 the necessary camera parameters and position the cameras in space ${ }^{31}$. Once a calibration 9 was performed the cameras were not moved relative to one another. A custom made 10 calibration plate (FIG. 3) of $2.4 \mathrm{~m} \times 2.4 \mathrm{~m}$ was made from an array of equally spaced 11 dots (10 mm diameter, $50 \mathrm{~mm}$ spacing) from 3 layer white black white sign material to 12 fill the area of interest. The calibration plate (consisting of rigidly joined two halves of $131.2 \mathrm{~m} \mathrm{x} 2.4 \mathrm{~m}$ ) placed on a floating $50 \mathrm{~mm}$ thick expanded polystyrene backing. The 14 surface of the calibration plate was measured to have floating elevation of $49 \mathrm{~mm}$ above 15 the still water level.
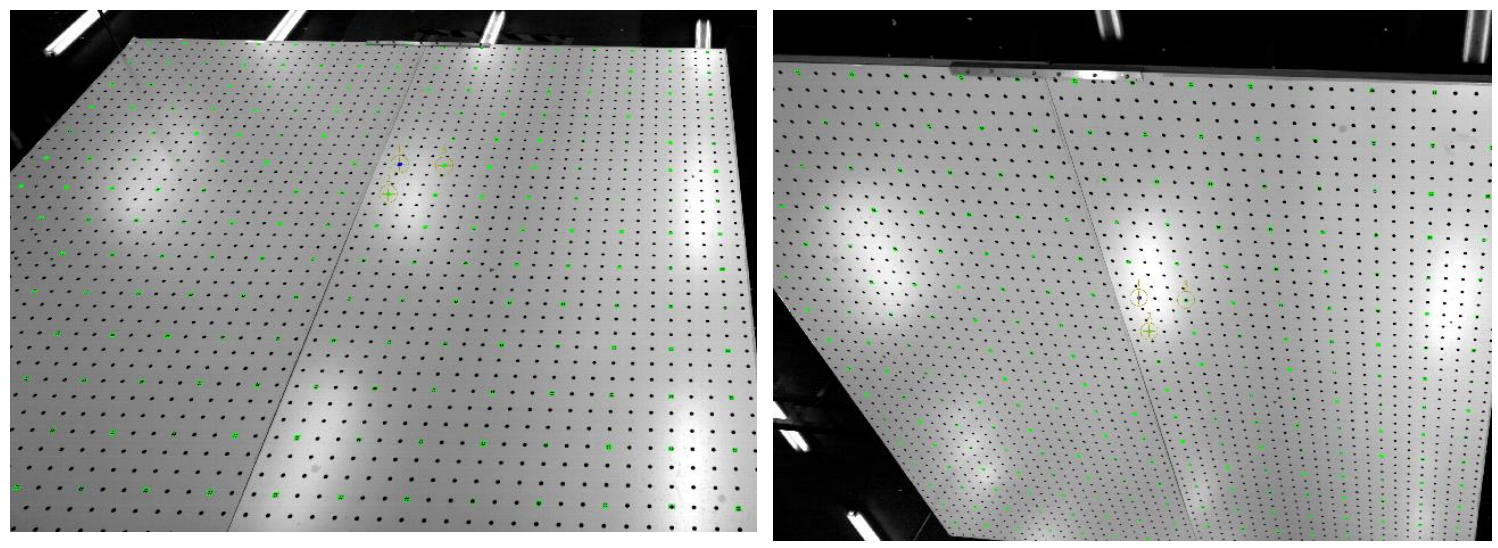

16 FIG. 3: View of calibration plate with selected calibration points and datum reference for 17 camera 1 (left) and camera 2 (right) (main camera set).

For the calibration to be valid it was necessary to acquire several "views" of the calibration plate at different planes throughout the area of interest. To perform this action the cameras were fitted on a cross-bar between two vertically oriented stepper motor driven linear stages. The cross-beam was then raised and lowered to provide a total of eight coplanar image pairs over a total vertical range of $170 \mathrm{~mm}$. The sum of the corrected images post-calibration (all views) from both camera sets is shown in FIG. 4 . Note that only every third calibration point was used for the first camera set and every second calibration point was used for the second camera set (green and white circles in

26 FIG. 3) which was found to provide lower overall calibration residuals (RMS of fit). 

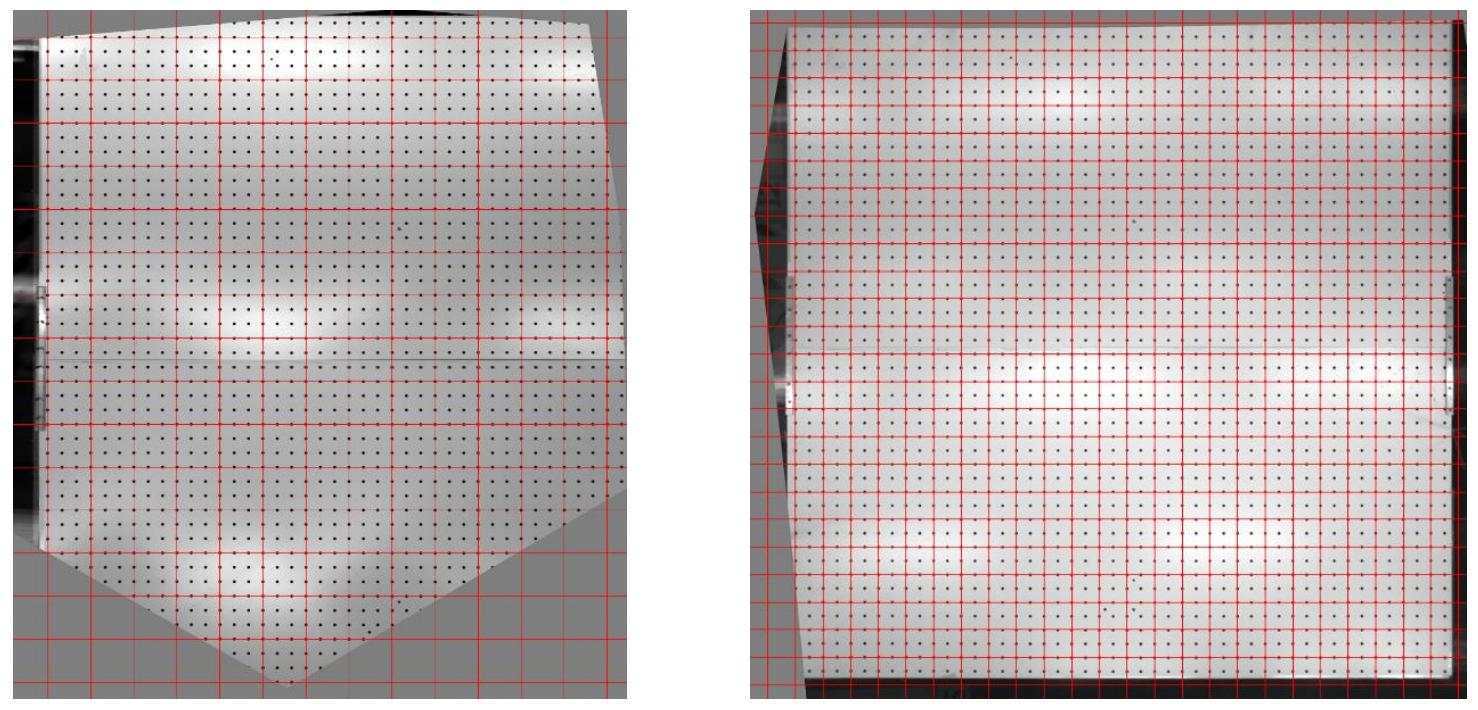

2 FIG. 4: Sum of corrected images from both cameras and all views. The grey border

3 signifies the extents of the camera overlap in the measurement volume. Left: Main

4 camera set, the red grid lines are $150 \mathrm{~mm}$ apart. Right: Secondary camera set, the red grid

5 lines are $100 \mathrm{~mm}$ apart.

In a more typical surface flow system; (consisting of cameras fitted with lenses having less distortion) the process described above would be sufficiently accurate for the software to reliably run to completion. However due to the requirement for a larger field

9 of view and the constraint on the object distance, the current lenses were selected. So in

10 the case for the data acquired; attempting to process the data resulted in a failed solution.

11 The cause of the failed solution was found to be that the calibration was inadequately

12 solved by the calibration wizard. To improve the calibration beyond that produced by the

13 standard calibration wizard, a software tool "self-calibration", available in a separate

14 DaVis 8.2 product Stereoscopic PIV, was used to improve the calibration. Not only did

15 the RMS of fit reduce from 0.66 pixels to 0.12 pixels for the main cameras but

16 importantly, the software ran more reliably. A summary of calibration details are

17 provided in Appendix A.

A measure of the accuracy of the calibration and DIC software can be evaluated

20 following sections.

\section{Image processing}

22 Image pairs were processed using the following settings as they were found to

23 give reliable results for the datasets throughout: subset size $=21$, step size $=10$,

24 calculation mode $=$ fast and maximum expected pixel displacement $=50$ pixels. 
The DIC software was validated by means of a vertical translation test during which 200 images were obtained whilst the cameras were traversed in the $Z$ direction from 0 to $100 \mathrm{~mm}$. Accuracy of the linear stage and its vertical alignment were not quantified with sufficient accuracy to state whether the source of error presented here was due to error in the photogrammetry system or misalignment of the linear translation system. FIG. 5 right shows the surface solution after the camera system was lowered a vertical displacement of $100 \mathrm{~mm}$ and the left image subtracted, the mean value is 99.7 $\mathrm{mm}$. Crudely this equates to an estimated uncertainty in the videogrammetry system of $90.3 \mathrm{~mm}$. The remainder surface (FIG. 5 right) is clearly not level, however examination 10 of the gradient (not shown) suggests the measured (still water) surface is flat so the error 11 can mostly be attributed to incorrect vertical alignment of the camera traverse system 12 rather than error in the stereo system or its calibration.

13
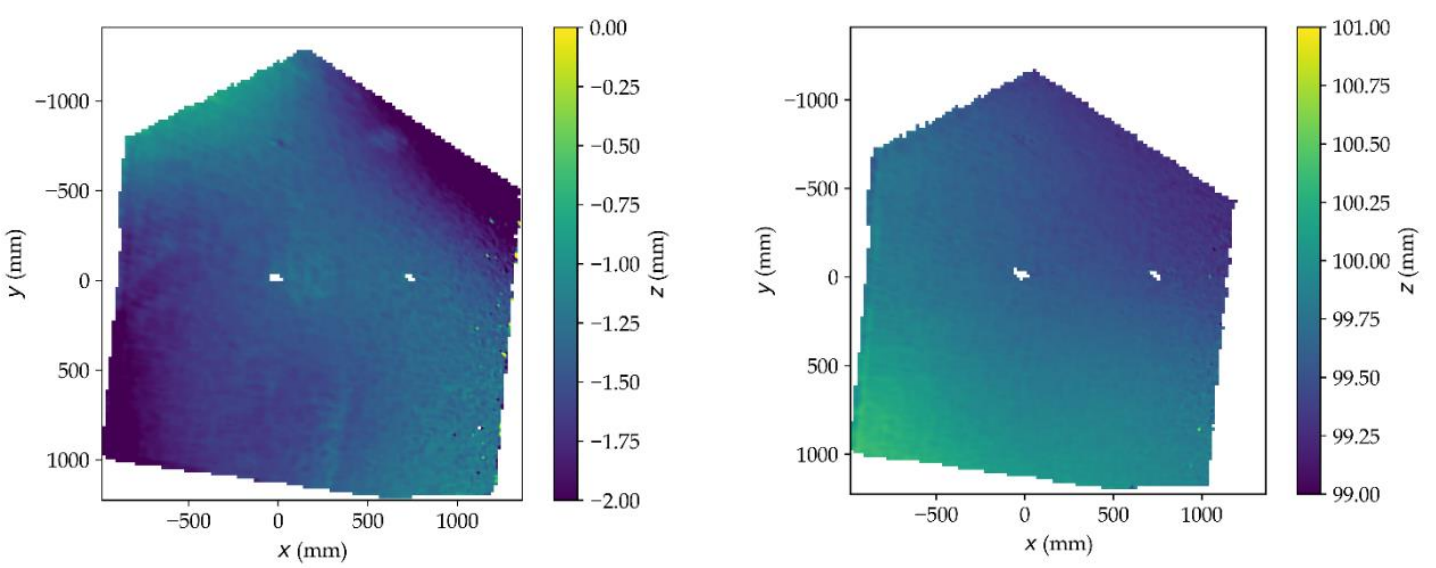

15 FIG. 5: Photogrammetry result from the still water surface after final calibration. Left: zero image. Right: $100 \mathrm{~mm}$ translation in $\boldsymbol{z}$ direction image with zero image subtracted.

\section{Bias error}

18 FIG. 5 left is an image of the still free surface after final calibration. It is clear 19 that not only is the surface not flat, the surface does not coincide with the zero plane. This

20 is partially attributed to calibration plates not being perfectly flat. In other types of data

21 acquisition systems it is typical to subtract a zero value, bias error, from the reading to 22 give the expected value ${ }^{32}$. A zero image was developed for each camera set using a 23 collection of images of the still free-surface as follows:

241 Each image decimated by a reduction factor of 2;

252 Each image resized by $3^{\text {rd }}$ order spline interpolation by a reduction factor of 6;

263 A median filter of size 20 is applied to each image;

274 Images averaged;

285 Missing data pixels expanded by a binary dilation of 10 pixels;

296 Missing data filled in using biharmonic inpaint (scikit-image inpaint); 
17 Image resized by $3^{\text {rd }}$ order spline interpolation by a growth factor of 12 to original 2 image size.

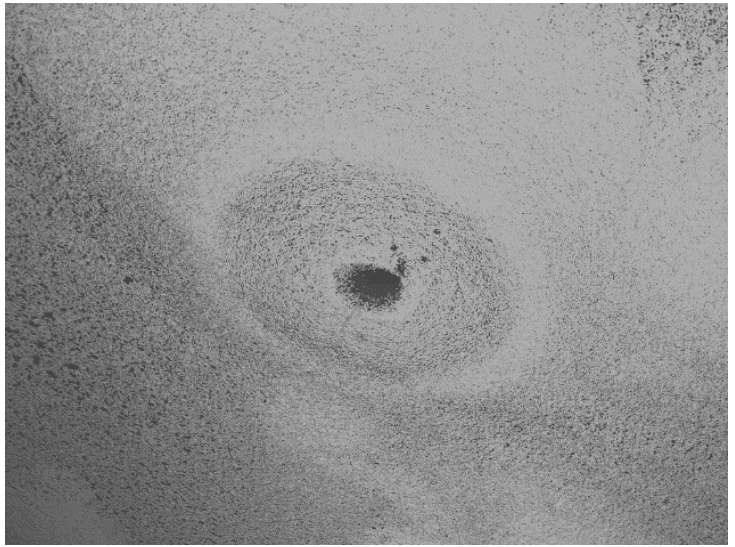

4 FIG. 6: Raw image from a main camera during an experiment for heaving sphere

5 showing particles are absent directly over the sphere location. Also a wave crest

6 generated by the heaving sphere is visible

$7 \quad$ Additionally, for the main camera set (considering the results for a heaving sphere 8 near the free surface), a circular region with a radius of $100 \mathrm{~mm}$ centred over the origin 9 was not able to be processed due to particles washing away from the region as a feature 10 of local currents caused by the sphere oscillation (FIG. 6). FIG. 7 shows both the zero 11 image (left) and an example of a corrected first frame (right) for the main and secondary 12 camera systems (top and bottom respectively). The remaining texture visible (FIG. 7 13 right) is a combination of physical texture present measured on the water surface due to 14 the presence of the fluorescent flakes, where some flakes may be sitting on top of others, 15 and residual uncertainty. Edge effects are introduced as a result of the filtering which is 16 most pronounced as can be seen by the yellow border in FIG. 7 lower right. The 17 remainder of the analysis presented will utilise data inside of the areas of the zero image 18 affected by the edge effect phenomena. 

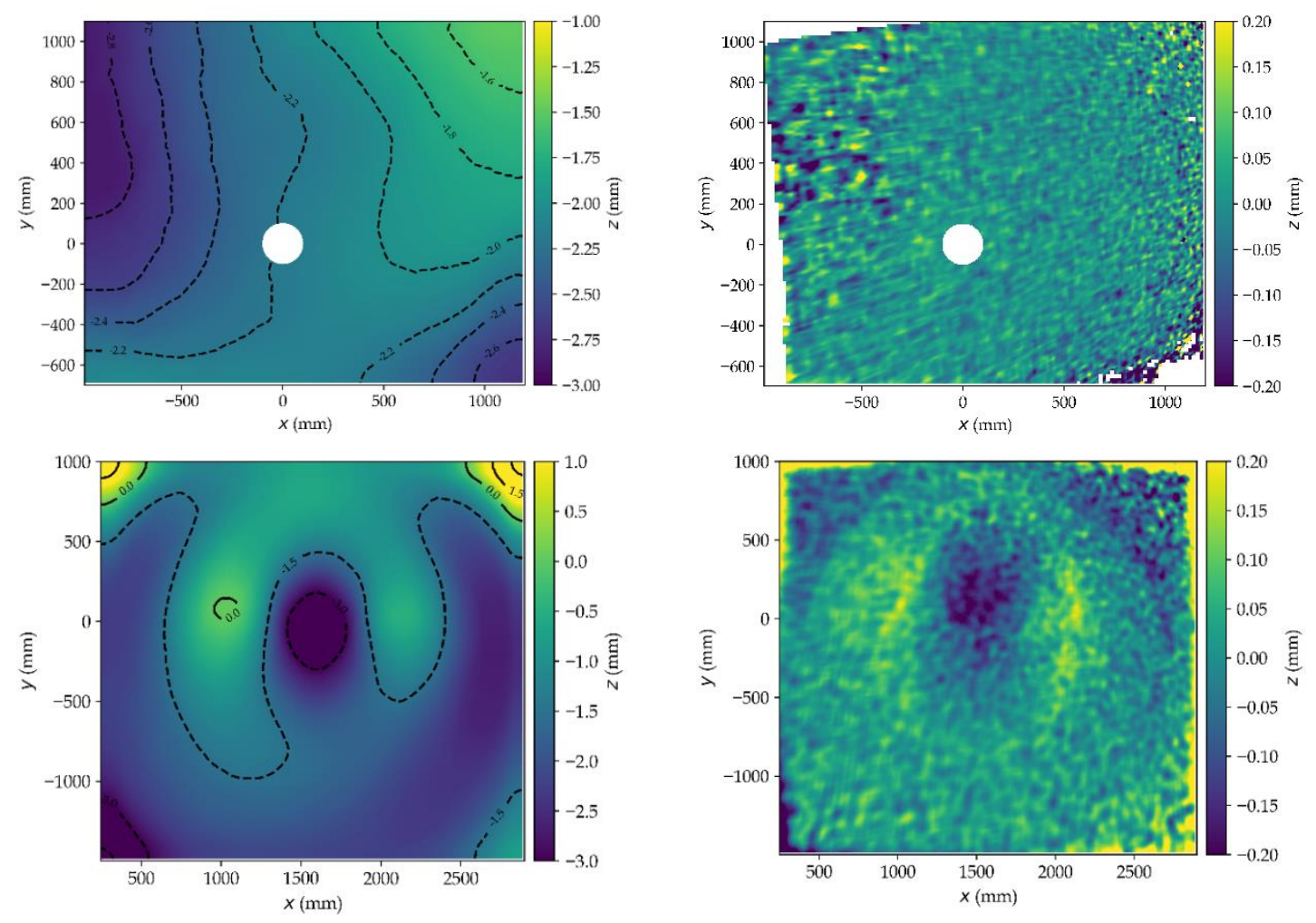

1 FIG. 7: Left: Calculated zero image to correct $\boldsymbol{Z}$ bias error. Right: Surface elevation of 2 the still water surface with the zero image (left) subtracted. Top row correspond to the

3 Main camera set and bottom row correspond to the secondary camera set.

\section{$4 \quad$ Surface velocity measurements}

In addition to surface elevation measurements it was possible to determine 3

6 dimensional surface displacement between frames, provided that image sequences are 7 taken with a sufficiently short time between frames. Ultimately this provides the user

8 with a velocity field of the surface, similar to Particle Imaging Velocimetry (PIV), but 9 returns the velocity for the surface rather than for a plane as is typical for PIV.

10 Preliminary results of surface flows have already been reported by the authors in ${ }^{29}$, 11 which was used to demonstrate the surface currents expected in the vicinity of wave 12 energy converters. Because the velocity is extracted from two sequential images, the 13 velocity is the average between the subsequent frames with an uncertainty in the timing 14 of the motion equal to the interframe time ( $40 \mathrm{~ms}$ in this case) centred on the midpoint 15 between the two images. Since the velocity fields are a differential result, it is not 16 necessary (or appropriate) to subtract a zero image to correct for bias error.

\section{Results}


Results are presented in this section for two experiments of a sphere oscillating vertically in sinusoidal motion for both one, and five complete cycles starting from the bottom of the stroke. The sphere oscillated with an amplitude of $70 \mathrm{~mm}$ and a frequency

4 of $1 \mathrm{~Hz}$. Sphere displacement is shown in FIG. 8. A ninth order $4 \mathrm{~Hz}$ low pass filter was 5 applied to the positional data to remove linear motor drive induced noise, $z=0 \mathrm{~mm}$ is 6 taken as the still water level.

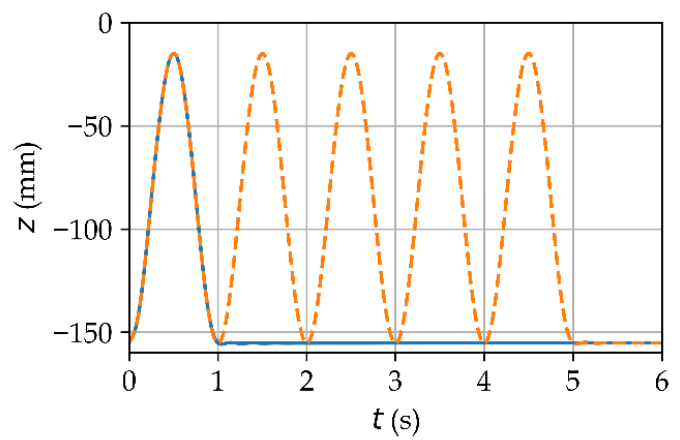

8 FIG. 8: Instantaneously position of the sphere top surface below the static water free 9 surface $(\mathrm{z}=0 \mathrm{~mm})$ where the blue solid line is a single oscillation and the dashed orange 10 line is five oscillations.

The surface radiation field for a single oscillation of the sphere is shown in FIG. 9 (using a reduced square window of the main camera set). The primary purpose of this set of images is to demonstrate the symmetry of the radiation field, but it is also interesting to note that a wave packet is generated by a single oscillation of the sphere which is thought to be caused by the proximity of the sphere to the free surface. Videos of the same are available as video 1 for a single oscillation and video 2 for five oscillations.

FIG. 10 : Left: is a plot of the upper and lower bounds of the free surface elevation radially extending out from the origin. Waviness in the appearance of the data is a feature of the rather low sampling frequency, while the discrepancy between neighbouring data points is a combination of measurement error and physical inconsistency in the radiated wave field. But clearly there is good agreement in data from different angles, which confirms the accuracy of the bias error correction through the use of a zero image.

FIG. 10 : Right: is a plot of the instantaneous surface amplitude from the Main and Secondary camera sets at the time instances of 1 second and 3.8 seconds sliced through $y=0$. There is good agreement between the two sets of data, however it is apparent that the Secondary camera set is smoother than the Main camera set which most notably reduces the amplitude of the measured crests and troughs of the steeper waves. The cause of the reduced accuracy of the Secondary camera set may be associated with the larger field of view, increased camera lens distortion (lower focal length lens) or slightly smaller pixel scale factor of $0.491 \mathrm{pixel} / \mathrm{mm}$ compared to $0.544 \mathrm{pixel} / \mathrm{mm}$ of the Main camera set. 

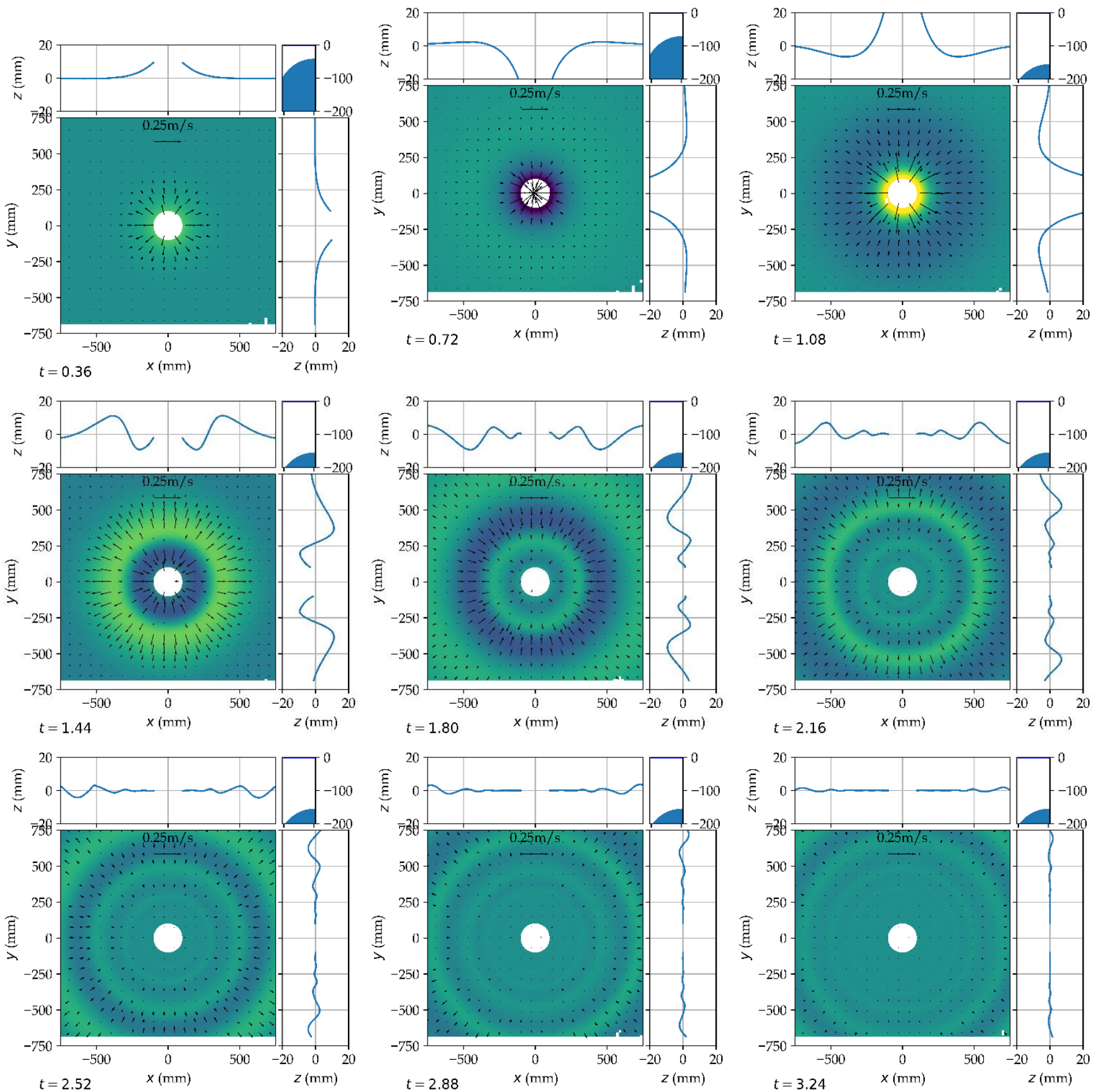

2 FIG. 9: Instantaneous water surface elevation and velocity generated by sphere heaving

3 for a single cycle at $1 \mathrm{~Hz}$ and $70 \mathrm{~mm}$ amplitude at time steps $\boldsymbol{t}=\mathbf{0 . 3 6}, \mathbf{0 . 7 2}, \mathbf{1 . 0 8}$,

$41.44,1.80,2.16,2.52,2.88,3.24$ from left to right and top to bottom

5 respectively. The centre white circle covers the fluid surface region where flakes were

6 missing due to the presence of surface currents. Instantaneous position of the sphere to

7 the free-surface is illustrated in the top right corner of each figure. 


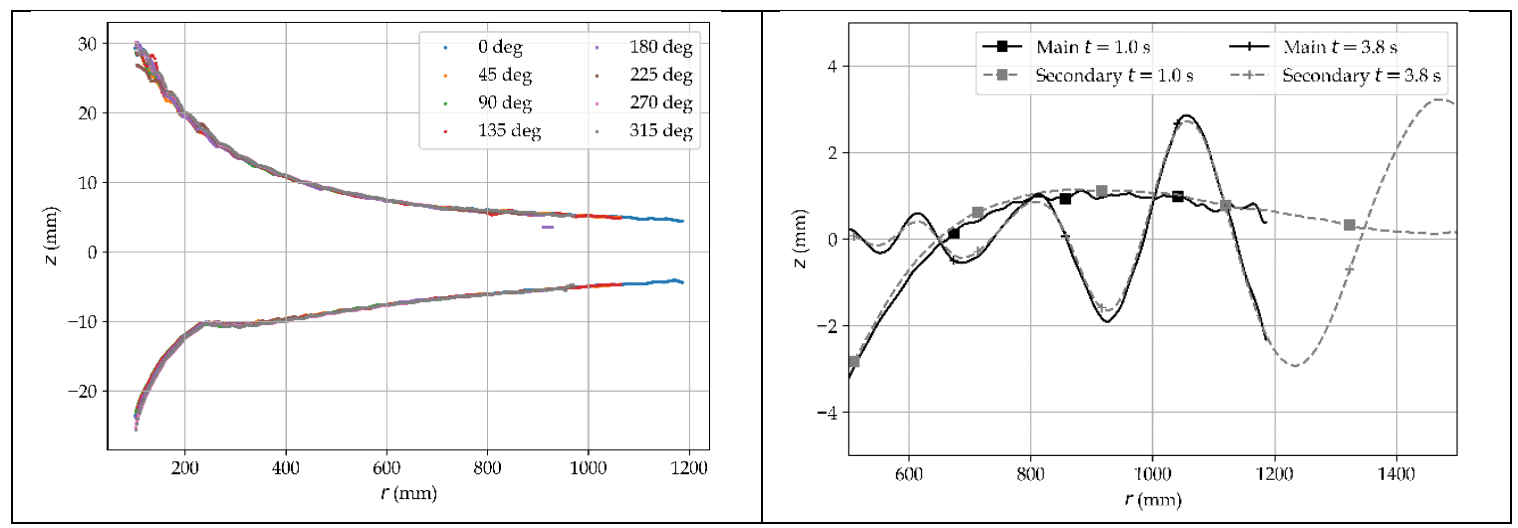

2 FIG. 10: Left: Radial maximum and minimum amplitudes at 45 degree intervals of 3 radiated wave field for a single sphere oscillation at $1 \mathrm{~Hz}$ and $70 \mathrm{~mm}$. Right:

4 Instantaneous surface profile at two different time instants for the Main and Secondary

5 camera sets sliced through $y=0$ for a single sphere oscillation at $1 \mathrm{~Hz}$ and $70 \mathrm{~mm}$.

The five cycle data set is used for an extended window to investigate validity of overlaying data from different camera sets. Image sets from both camera sets were

8 combined on a frame by frame basis by first resizing the images to the full sized window

9 using third order spline interpolation ( $2 \mathrm{~mm}$ spacing between pixels). Images from the

10 main and secondary sets were then averaged using a weighted average technique, which

11 generated a weighting image using Gaussian blur applied to a unity array (ones) of equal

12 shape to the base image. FIG. 11 is a sequence of time-series snapshots of surface profile

13 taken through the centreline at $y=0$. The overlap between the two sets of data occurs at 14 around $800 \mathrm{~mm}$ from which there is no obvious discontinuity in the profile. 

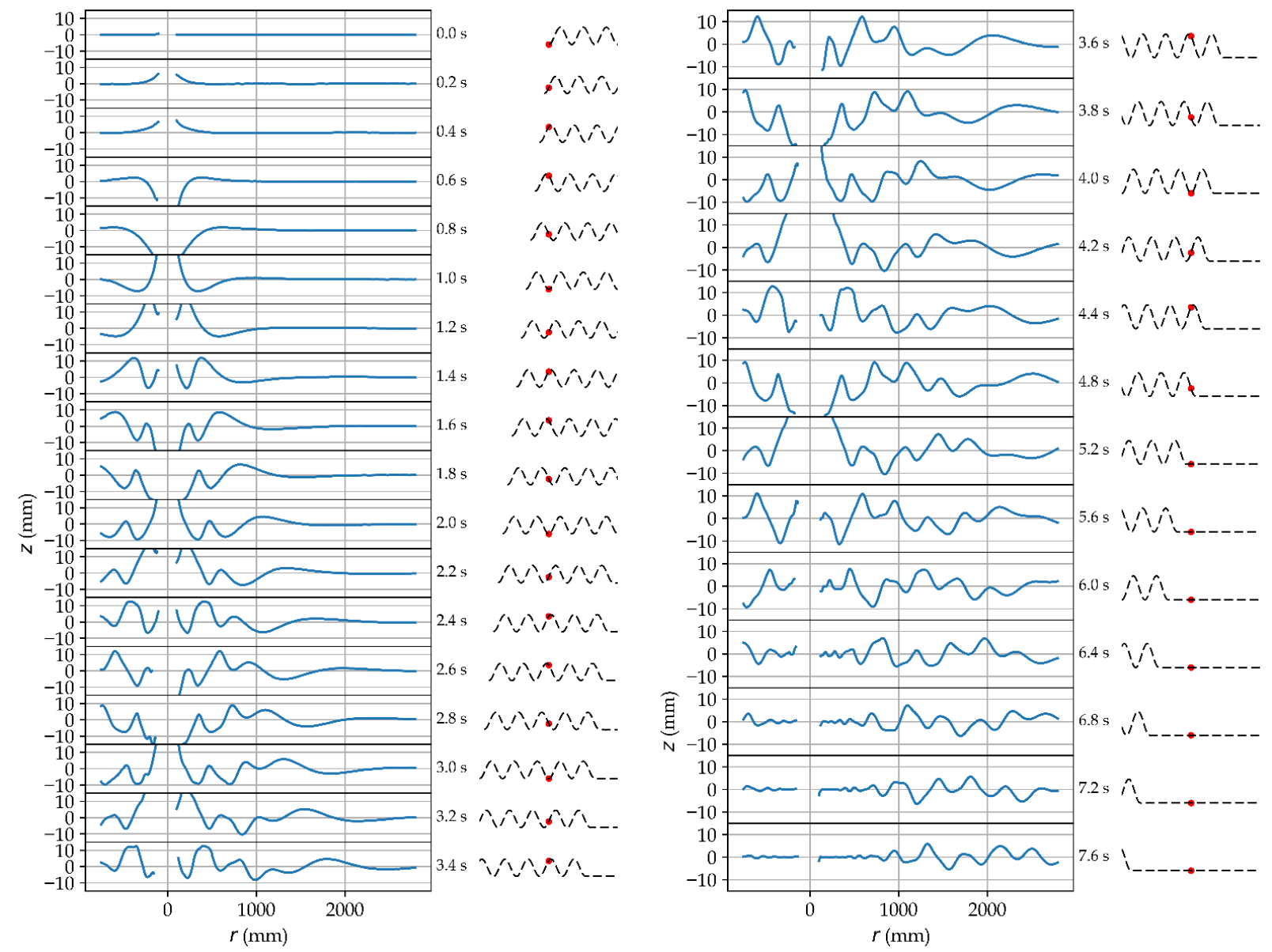

2 FIG. 11: Cross-section of surface profile time-series of wave field generated by sphere 3 heaving at $1 \mathrm{~Hz}$ and $70 \mathrm{~mm}$ amplitude (5 complete cycles) composed of the merged 4 surface profile from the main and secondary camera sets. The dashed line indicates the 5 path history of the sphere and the red dot indicates the location of the sphere at that time 6 instant.

Given that there is periodic motion in this data set; it is possible to apply periodic 8 analysis to the time-series data such as phase-averaging or spectral analysis. In this case a 9 spectral analysis was chosen for two reasons. First, the harmonic components and phase 10 are both obtained. Second, only two cycles of data were available containing the 11 developed flow over the majority of the region of interest. This would provide an 12 inadequate amount of samples for phase-averaging to be effective. Spectral analysis using 13 Fourier Transforms is less sensitive to the number of cycles, rather, the total number of 14 samples only affects the Nyquist frequency.

Spectral analysis was performed on a pixel by pixel basis (similar to a method described in Longo and Stern $\left.{ }^{33}\right)$ for the same data set used to generate FIG. $11(y=0)$ but limited to the time $3 \leq t \leq 5$ seconds giving 51 data points per pixel. Using $3^{\text {rd }}$ order spline interpolation, data was resampled to $2^{n}=256$ data points $(n=8)$ to prevent zero padding during Fast Fourier Transform analysis. First, second and third harmonics were 
then calculated corresponding to the original timestamps using $\eta_{n}=$

$2 A_{n} \cos \left(2 \pi n \phi_{1}\left(t-t_{0}\right)+\phi_{n}\right)$, where $\eta_{n}$ is the instantaneous profile at time $t$ relative to

3 starting time $t_{0}$, and $\phi_{n}$ is the phase of the $n$th harmonic.

FIG. 12 is a plot of the instantaneous profile corresponding to $t=3.28 \mathrm{~s}$. Both the original data and summed harmonic components are plotted so we can evaluate the effectiveness to reconstruct the original wave profile. The original profile at $t=3.28 \mathrm{~s}$ is plotted as a solid black line and the original data from $t=4.28 \mathrm{~s}$ is plotted as a dashed grey line. The data points of the measured data correspond well for radius values between $r=200 \mathrm{~mm}$ and $r=1000 \mathrm{~mm}$, after which there is some divergence that is attributed to the third order component not having propagated past the $1000 \mathrm{~mm}$ radius. The sum of the first three harmonics are plotted as a dotted blue line. In this case the harmonic analysis is shown to be effective for the region $400<r<1000 \mathrm{~mm}$. The reason for the deviation of the fit from the data for radius $r<400 \mathrm{~mm}$ is due to the proximity of the sphere to the free-surface. Whereas the cause of deviation for radius $r>$ $1000 \mathrm{~mm}$ was due to differing wave profiles in the source data (absence of the third harmonic as mentioned above). The time and distance for the propagation of the third order harmonic wave component (propagating at $C_{g}=g / 4 \pi f$ ) can be predicted with linear wave theory which equates to $780 \mathrm{~mm}$ (for $t=3 \mathrm{~s}$ ), and if the diameter of the sphere is taken into consideration this appears to agree with that observed. In short, the origin of the wave front should not necessarily be assumed to be $r=0$. An animation of the same data is provide in video3.

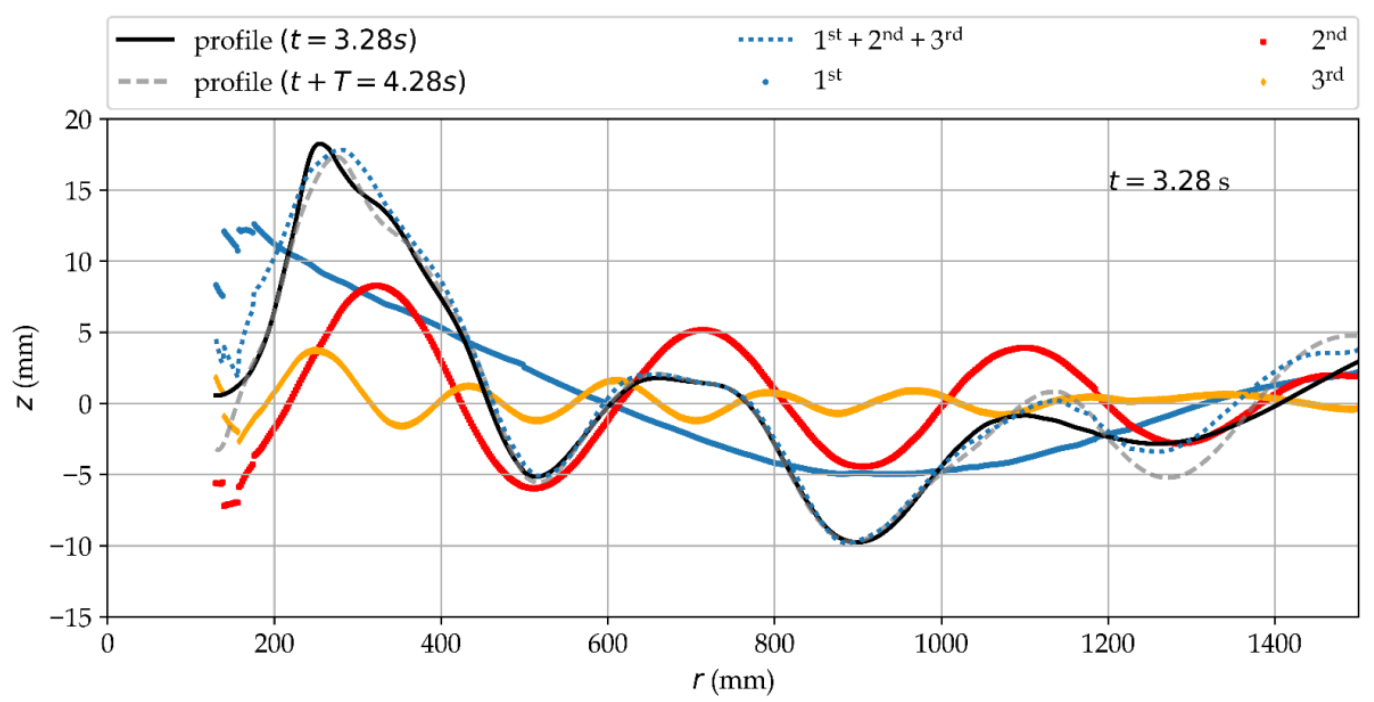

FIG. 12: Profiles of the actual surface (black solid and then grey dashed for $\boldsymbol{t}+\boldsymbol{T}$ ) and reconstructed water surface (blue dots) of wave field generated by the sphere heaving at 1 $\mathrm{Hz}$ and $70 \mathrm{~mm}$ amplitude at equvalent time $\boldsymbol{t}=\mathbf{3 . 2 8} \mathrm{s}$. First, second and third order reconstructed surface are added as blue circles, orange squares, and orange diamonds respectively.

Furthermore, it was possible to analyse the individual frequency components of the data from the previous section to extract wavelength and celerity. Firstly, the 
1 wavelength was directly measured and averaged from the frames available. Secondly, 2 wave celerity was calculated from the measured displacement of wave crests between 3 respective frames divided by the time between frames. Results are summarised and 4 compared to those predicted through linear theory in Table 1, agreement is within two 5 standard deviations of the measured values.

6 Table 1 : Wavelength and celerity of harmonic components of wave field generated by

7 sphere heaving at $1 \mathrm{~Hz}$ and $70 \mathrm{~mm}$ amplitude measured from Fourier analysis and

8 compared against deepwater linear wave theory.

\begin{tabular}{cl|c|c|c} 
& \multicolumn{2}{c|}{ Wavelength $(\mathrm{m})$} & \multicolumn{2}{c}{ Celerity $(\mathrm{m} / \mathrm{s})$} \\
\cline { 2 - 5 } Harmonic & Experimental & Linear theory & Experimental & Linear theory \\
\hline 1 & $1.477 \pm 0.015$ & 1.561 & $1.488 \pm 0.143$ & 1.56 \\
2 & $0.389 \pm 0.005$ & 0.390 & $0.781 \pm 0.059$ & 0.78 \\
3 & $0.187 \pm 0.023$ & 0.173 & $0.474 \pm 0.062$ & 0.52
\end{tabular}

\section{Velocity field generated by heaving sphere}

13 As mentioned previously, the instantaneous velocity fields are calculated from 14 adjacent image pairs, thus the time instant of the motion is averaged between the two 15 images. This also means that the most likely time instant of the displacement (with the 16 assumption of constant velocity between two images) occurs at the time instant between 17 the two images. To approximately account for time offset the velocity fields shown in 18 FIG. 9 are interpolated with $3^{\text {rd }}$ order splines from adjacent velocity fields to closer 19 represent the time instant at which the surface elevation was measured.

Utilising velocity field data instead of surface elevation FIG. 13 is similar to FIG.

2110 but is a scatter of the maximum surface particle velocity in that data set rather than elevation. The figure clearly demonstrates that wave field velocity is axisymmetric and also that the maximum particle velocities diminish with increasing radius. Close to the source of the wave there are a scatter of lower magnitude data points reflecting the issue previously highlighted regarding floating markers washing away due to the upwelling water associated with the sphere's motion. 


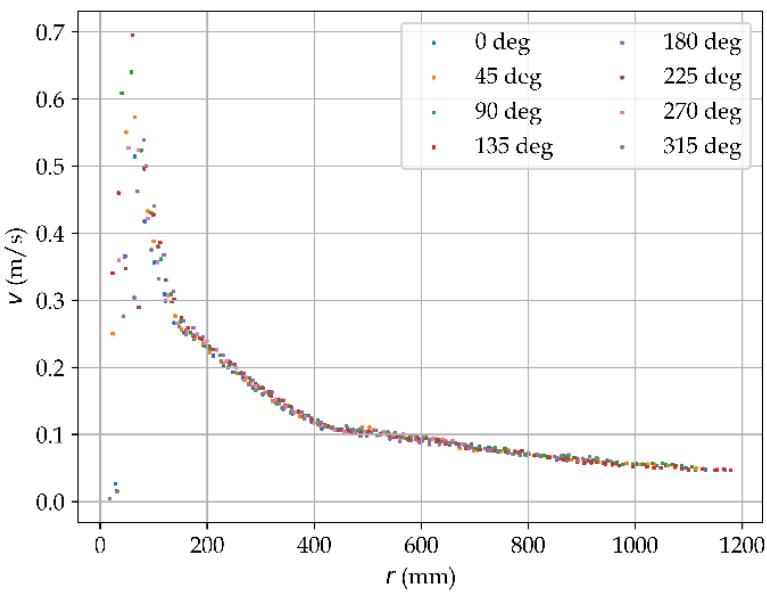

2 FIG. 13: Radial maximum surface particle velocity at 45 degree intervals of radiated

3 wave field for a single sphere oscillation heaving at $1 \mathrm{~Hz}$ and $70 \mathrm{~mm}$.

\section{Conclusions}

Spatial measurement of the water surface in hydrodynamic test facilities will continue to be an area of interest to researchers. In this paper it was demonstrated that it is feasible to utilise DIC software in conjunction with floating fluorescent surface

8 markers to provide good surface elevation and velocity measurements over a

9 considerable area of $1.5 \mathrm{~m} \times 1.5 \mathrm{~m}$. The field of view may be extended through the use of

10 additional stereo camera pairs with no particular complication.

Acquisition of high resolution surface measurements enables feature extraction otherwise unobtainable through point measurements, such as instantaneous snapshots of free-surface elevation, true measurement of wavelength, wave group celerity and the surface particle velocity field. Imaging based systems will continue to require good quality artefact free images, advancements might be possible with hardware improvements including noise reduction and corresponding sensitivity.

The main limitation found in application of this method was imaging of the free surface. Utilisation of floating surface markers was found to be effective for cases where station keeping of the floating markers was possible and no inflow sources (without markers) exist, typical for irrotational flow such as low steepness non-breaking waves. Usefulness of the method can be immediately extended through proper acquisition of stereo image pairs of a textured water surface.

\section{Acknowledgements}

We thank the following for their assistance in experimental setup and data 25 acquisition throughout the project: Guy McCauley, Jeremy Ledoux, Romain Briand, Kirk

26 Meyer and Liam Honeychurch. We also thank staff at LaVision for their technical

27 support in initial processing of the data and comments on how to improve processing 28 reliability. 


\section{Funding}

2

3

4

5

6

This work was supported by the Australian Renewable Energy Agency Emerging Renewables Program [ERP A00575 - Towards an Australian capability in arrays of ocean wave-power machines]. Brian Winship was jointly funded by CSIRO Oceans and Atmosphere Climate Research Centre and the Australian Renewable Energy Agency (ARENA) Emerging Renewables Program (ERP A00521 - The Australian Wave Energy Atlas Project).

\section{References}

1. Payne GS, Taylor J, Ingram D. Best practice guidelines for tank testing of wave energy converters. The Journal of Ocean Technology 2009; 4: 38-70.

2. Zavadsky A, Benetazzo A, Shemer L. On the two-dimensional structure of short gravity waves in a wind wave tank. Physics of Fluids 2017; 29: 016601.

3. Stratigaki V. Experimental study and numerical modelling of intra-array interactions and extra-array effects of wave energy converter arrays. Dissertation, Ghent University, http://hdl.handle.net/1854/LU-5664337 (2014, accessed 9 February 2017).

4. Fleming A, Penesis I, Goldsworthy L, et al. Phase averaged flow analysis in an oscillating water column wave energy converter. Journal of Offshore Mechanics and Arctic Engineering 2012; 135: 021901-[1-9].

5. O'Boyle L, Elsäßer B, Whittaker T. Experimental measurement of wave field variations around wave energy converter arrays. Sustainability 2017; 9: 70.

6. Rak G, Hočevar M, Steinman F. Measuring water surface topography using laser scanning. Flow Measurement and Instrumentation 2017; 56: 35-44.

7. Blenkinsopp CE, Turner IL, Allis MJ, et al. Application of LiDAR technology for measurement of time-varying free-surface profiles in a laboratory wave flume. Coastal Engineering 2012; 68: 1-5.

8. Gomit G, Chatellier L, Calluaud D, et al. Large-scale free surface measurement for the analysis of ship waves in a towing tank. Exp Fluids 2015; 56: 184.

9. Bergamasco F, Torsello A, Sclavo M, et al. WASS: An open-source pipeline for 3D stereo reconstruction of ocean waves. Computers \& Geosciences 2017; 107: 28-36.

10. Moisy F, Rabaud M, Salsac K. A synthetic Schlieren method for the measurement of the topography of a liquid interface. Exp Fluids 2009; 46: 1021.

11. Damiano AP, Brun P-T, Harris DM, et al. Surface topography measurements of the bouncing droplet experiment. Exp Fluids 2016; 57: 163. 
12. Engelen L, Creëlle S, Schindfessel L, et al. Spatio-temporal image-based parametric water surface reconstruction: a novel methodology based on refraction. Meas Sci Technol 2018; 29: 035302.

13. Hamachi S, Sanada Y, Toda Y. A technique to measure wave height distributions by the reflected light image. Journal of the Visualization Society of Japan 2006; 26: $17-$ 20.

14. Aureli F, Dazzi S, Maranzoni A, et al. A combined colour-infrared imaging technique for measuring water surface over non-horizontal bottom. Exp Fluids 2014; 55: 1701.

15. Chatellier L, Jarny S, Gibouin F, et al. A parametric PIV/DIC method for the measurement of free surface flows. Exp Fluids 2013; 54: 1488.

16. Tamburrino A, Gulliver JS. Free-surface visualization of streamwise vortices in a channel flow. Water Resour Res 2007; 43: W11410.

17. Kumar S, Gupta R, Banerjee S. An experimental investigation of the characteristics of free-surface turbulence in channel flow. Physics of Fluids 1998; 10: 437-456.

18. Dabiri D, Gharib M. Simultaneous free-surface deformation and near-surface velocity measurements. Experiments in Fluids 2001; 30: 381-390.

19. Turney DE, Anderer A, Banerjee S. A method for three-dimensional interfacial particle image velocimetry (3D-IPIV) of an air-water interface. Meas Sci Technol 2009; 20: 045403.

20. Sokoray-Varga B, Józsa J. Particle tracking velocimetry (PTV) and its application to analyse free surface flows in laboratory scale models. Periodica Polytechnica Civil Engineering 2008; 52: 63-71.

21. Wanek JM, Wu $\mathrm{CH}$. Automated trinocular stereo imaging system for threedimensional surface wave measurements. Ocean Engineering 2006; 33: 723-747.

22. Benetazzo A, Fedele F, Gallego G, et al. Offshore stereo measurements of gravity waves. Coastal Engineering 2012; 64: 127-138.

23. Ferreira E, Chandler J, Wackrow R, et al. Automated extraction of free surface topography using SfM-MVS photogrammetry. Flow Measurement and Instrumentation 2017; 54: 243-249.

24. Schanz D, Gesemann S, Schröder A. Shake-The-Box: Lagrangian particle tracking at high particle image densities. Exp Fluids 2016; 57: 70.

25. Kiefhaber D, Caulliez G, Zappa CJ, et al. Water wave measurement from stereo images of specular reflections. Meas Sci Technol 2015; 26: 115401. 
1 26. Gomit G, Chatellier L, Calluaud D, et al. Free surface measurement by stereorefraction. Exp Fluids 2013; 54: 1540.

3 27. Tauro F, Porfiri M, Grimaldi S. Fluorescent eco-particles for surface flow physics $4 \quad$ analysis. AIP Advances 2013; 3: 032108.

5 28. Penesis I, Manasseh R, Nader J-R, et al. Performance of ocean wave-energy arrays in Australia. In: Proceedings of the 3rd Asian Wave \& Tidal Energy Conference, 24 28 October 2016. Singapore, pp. 246-253.

8 29. Fleming A, Manasseh R. Experimental observation of surface currents produced by WEC radiation and diffraction. In: Lewis A (ed) Proceedings of the Twelfth European Wave and Tidal Energy Conference. University College Cork, Ireland: EWTEC, 2017, pp. 802-1-802-7.

30. Nader J-R, Fleming A, Macfarlane G, et al. Novel experimental modelling of the hydrodynamic interactions of arrays of wave energy converters. International Journal of Marine Energy 2017; 20: 109-124.

15 31. LaVision. Flowmaster - product manual for DaVis 8.3. Anna-Vandenhoeck-Ring 19, D-37081 Göttingen: LaVision, 2016.

32. ITTC. Guide to the expression of uncertainty in experimental hydrodynamics, ITTC guide 7.5-02-01-01, revision 01. 2008.

33. Longo J, Stern F. Uncertainty assessment for towing tank tests with example for surface combatant DTMB model 5415. Journal of ship research 2005; 49: 55-68. 


\section{Appendix A}

2 Table 2: Calibration factors for surface flow calibration using a software wizard standard

3 pinhole calibration and the stereo PIV "self-calibration"

\begin{tabular}{|c|c|c|c|c|c|c|c|c|c|c|}
\hline \multirow{3}{*}{$\begin{array}{l}\text { Camera set } \\
\text { Calibration }\end{array}$} & & & \multicolumn{4}{|c|}{ Main (Basler Beat) } & \multicolumn{4}{|c|}{ Secondary (Basler Ace) } \\
\hline & & & \multicolumn{2}{|c|}{$\begin{array}{c}\text { Standard } \\
\text { Pinhole calibration }\end{array}$} & \multicolumn{2}{|c|}{$\begin{array}{c}\text { Final } \\
\text { Pinhole calibration }\end{array}$} & \multicolumn{2}{|c|}{$\begin{array}{c}\text { Standard } \\
\text { Pinhole calibration }\end{array}$} & \multicolumn{2}{|c|}{$\begin{array}{c}\text { Final } \\
\text { Pinhole calibration }\end{array}$} \\
\hline & & & Camera 1 & | Camera 2 & Camera 1 & Camera 2 & Camera 1 & Camera 2 & Camera 1 & Camera 2 \\
\hline RMS of fit (pixels) & & & \multicolumn{2}{|c|}{0.66308} & 0.11614 & 0.12424 & \multicolumn{2}{|c|}{0.52474} & 0.10170 & 0.11274 \\
\hline \multicolumn{2}{|l|}{ Camera configuration } & $\begin{array}{l}\text { Focal length }(\mathrm{mm}) \\
\text { Pixel Size }(\mathrm{mm}) \\
\text { Pixel aspect ratio }\end{array}$ & \multicolumn{2}{|c|}{$\begin{array}{c}24.436 \\
0.011 \\
1\end{array}$} & \multicolumn{2}{|c|}{$\begin{array}{c}24.436 \\
0.011 \\
1\end{array}$} & \multicolumn{2}{|c|}{$\begin{array}{c}9.1471 \\
0.0055 \\
1\end{array}$} & \multicolumn{2}{|c|}{$\begin{array}{c}9.20065 \\
0.0055 \\
1 \\
\end{array}$} \\
\hline \multirow[t]{6}{*}{ Calibration plate position $(\mathrm{z}=0 \mathrm{~mm})$} & Translation & $\mathrm{Tx}(\mathrm{mm})$ & -794.621 & -794.621 & -800.161 & -754.183 & -277.546 & 261.3 & -1071.22 & -546.302 \\
\hline & & Ty $(\mathrm{mm})$ & 259.202 & 259.202 & 265.417 & -72.8042 & 395.249 & 343.449 & 76.6162 & -75.9249 \\
\hline & & $\mathrm{Tz}(\mathrm{mm})$ & 2786.72 & 2786.72 & 2768.65 & 2452.2 & 2367.79 & 2335.27 & 2696.03 & 2280.31 \\
\hline & Rotation & Rx (deg) & -4.97765 & -4.97765 & -4.5612 & -3.80478 & -15.4652 & -13.1571 & -15.2976 & -13.5028 \\
\hline & & Ry (deg) & 45.7358 & 45.7358 & 45.6992 & -40.9343 & 14.0031 & -12.1781 & 14.4464 & -12.1141 \\
\hline & & Rz (deg) & -97.313 & -97.313 & -96.8922 & -92.7693 & -4.40905 & 2.7473 & -3.49725 & 3.77372 \\
\hline Size of dewarped image & & & \multicolumn{2}{|c|}{$1273 \times 1911$} & \multicolumn{2}{|c|}{$1275 \times 1925$} & \multicolumn{2}{|c|}{$2777 \times 2817$} & \multicolumn{2}{|c|}{$1844 \times 1902$} \\
\hline Camera scale & origin & $\begin{array}{l}\mathrm{x}_{0} \text { (pixel) } \\
\mathrm{y}_{0} \text { (pixel) } \\
\text { Scale factor (pixel/mm) }\end{array}$ & \multicolumn{2}{|c|}{$\begin{array}{c}536.733 \\
684.59\end{array}$} & \multicolumn{2}{|c|}{$\begin{array}{c}534.733 \\
691.59 \\
0.544092\end{array}$} & \multicolumn{2}{|c|}{$\begin{array}{c}1348.35 \\
765.61 \\
0.742396\end{array}$} & \multicolumn{2}{|c|}{$\begin{array}{c}504.64 \\
708.69 \\
0.491319\end{array}$} \\
\hline \multirow[t]{4}{*}{ Image distortion } & Principal point & $x_{p}(p x)$ & 1700.68 & 1700.68 & 1702.18 & 1707.18 & 1018.11 & 1011.35 & 1015.37 & 1014.59 \\
\hline & & $y_{p}(p x)$ & 782.157 & 782.157 & 779.945 & 812.495 & 1072.82 & 1062.08 & 1071.88 & 1058.79 \\
\hline & Radial distortion & $1^{\text {st }}$ order & 0.997144 & 0.997144 & 0.062119 & 0.87402 & 11.3782 & 9.86013 & 8.40527 & 8.34727 \\
\hline & & $2^{\text {nd }}$ order & 0 & 0 & 0.286693 & | -0.00198 & | -0.94744 & |-0.33996 & 0.078699 & 0.229095 \\
\hline
\end{tabular}

